\title{
Pelvis and Groin
}

\author{
Practical Anatomy, Injury Patterns, and Imaging \\ Findings
}

Robert D. Boutin (1) and Philip Robinson (1)

\section{Learning Objectives}

- At the anterior aspect of the pelvis, review groin pain in athletes, including appropriate imaging workup, terminology, and injury patterns.

- At the lateral aspect of the pelvis, highlight the key anatomy and imaging findings associated with abductor tendon disorders and proximal iliotibial band syndrome.

- At the inferior aspect of the pelvis, highlight the key anatomy and imaging findings associated with hamstring disorders and ischiofemoral impingement.

- At the posterior aspect of the pelvis, highlight the key anatomy and imaging findings associated with injuries at the sacrum and coccyx.

The groin and pelvis represent a large anatomical region with disorders affecting a diverse array of osteoarticular, musculotendinous, gastrointestinal, and genitourinary structures. Clinically, it can be difficult to pinpoint a pain generator, and imaging often proves helpful for establishing a diagnosis - particularly if the imager is familiar with practical anatomy, common injury patterns, and high-yield imaging findings.

Anatomy is fundamental to understanding the key concepts in this chapter (Figs. 6.1 and 6.2). The groin and pelvis

\section{R. D. Boutin $(\square)$}

Department of Radiology, Stanford University School of Medicine,

Stanford, CA, USA

e-mail: boutin@stanford.edu

P. Robinson

Musculoskeletal Centre X-Ray Department, Leeds Teaching

Hospitals Trust, Chapel Allerton Hospital, Leeds, UK

NIHR Leeds Biomedical Research Centre, Leeds Teaching

Hospitals, NHS Trust, Leeds, UK

e-mail: Philip.robinson10@nhs.net are at the junction of the trunk and lower extremities and are subjected to prodigious compression, traction, and shearing forces. For example, even with an everyday activity like a "single leg stance," physiologic loading can be phenomenal: $\sim 7.7$ times the body weight $(4653 \mathrm{~N})$ [1]. In athletes, powerful muscle contractions and various "supra-physiologic" demands can cause biomechanical overloading from acute trauma or repetitive microtrauma.

Radiologists benefit from knowledge of anatomic target sites for biomechanical failure and common injury patterns. Injuries can occur across a life span in acute, chronic, and acute-on-chronic settings. While stress fractures can occur in both pediatric and adult patients, there are other types of injuries favoring particular age groups.

- In skeletally immature patients, a frequent "weak link" in the "kinetic chain" is the apophyseal physis (which is weaker than the adjacent bone and tendon). Apophyseal avulsions in the pelvis most commonly occur at the ischial tuberosity and anterior superior iliac spine; other characteristic sites include the anterior inferior iliac spine, iliac crest, and pubic symphysis (Figs. 6.3 and 6.4). Of note, prior to ossification of apophyses (e.g., typically from 13 to 15 years of age at the ischial tuberosity), avulsions are occult radiographically [2]. Apophysitis also can occur in this age group [3].

- In young adults, the myotendinous junction is a common point of biomechanical failure for acute non-contact injuries [4]. Particularly frequent sites of injury in young adult athletes include the myotendinous junction of the hamstrings and rectus femoris (Figs. 6.5-6.7). With repetitive microtrauma, often with muscle imbalances, overuse injuries often occur at the tendon (tendinopathy) with peritendinous edema (e.g., peritendinitis, enthesitis).

- In older adults, tendon degeneration is increasingly prevalent, with resultant weakening of the tendon. Imaging commonly showing tendinopathy and tendon tearing. 

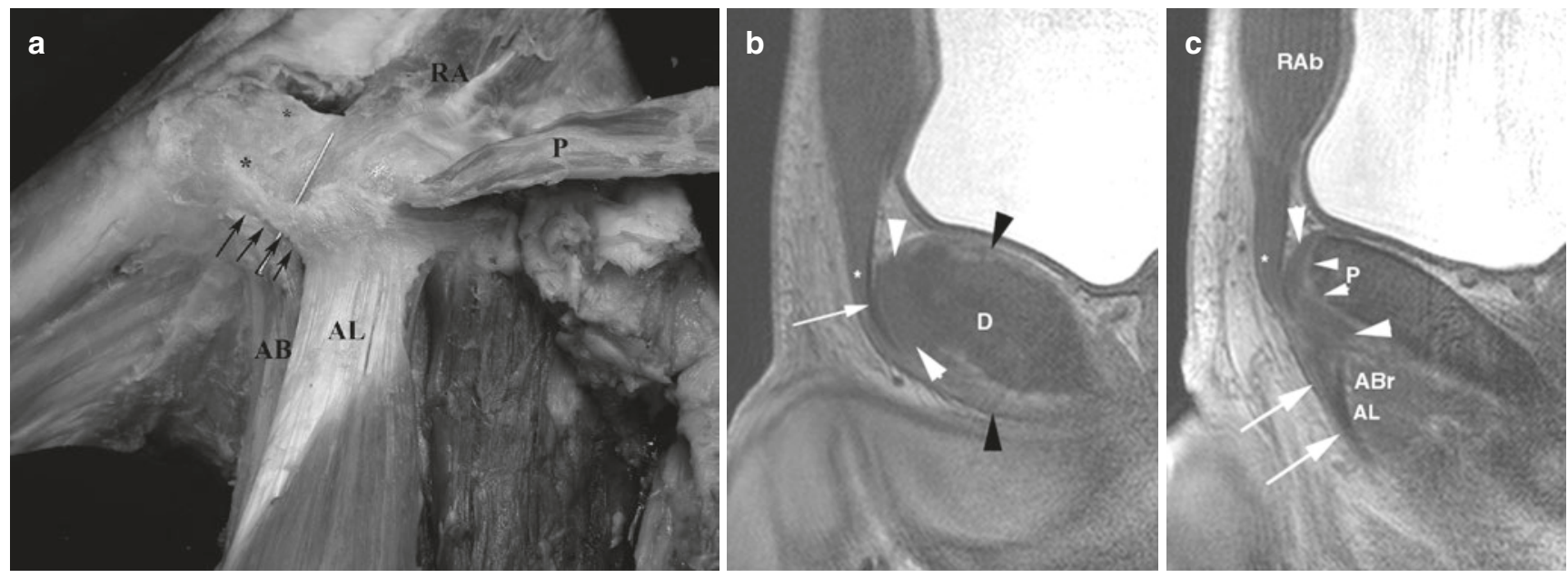

Fig. 6.1 (a) Normal anatomy, male cadaver dissection, anterolateral view. Asterisks mark the center of the symphysis pubis. The tendinous attachment of adductor longus (AL) to tissue overlying the anterior symphysis pubis is elevated by a pin and inferiorly outlined with arrows. Pyramidalis (P) is reflected to reveal rectus abdominis (RA). The medial margin of adductor brevis $(\mathrm{AB})$ can be seen medial to adductor longus (AL). (b) Normal sagittal MRI anatomy. A 29-year-old male athlete (experienced previous pubalgia). Sagittal T1 FFE MR image shows edge of fibrocartilagenous disk (D), interdigitating hyaline cartilage and pubic bone (black arrowheads). Anteriorly, the capsular tissues (white arrowheads) merge with the disk (D) and rectus

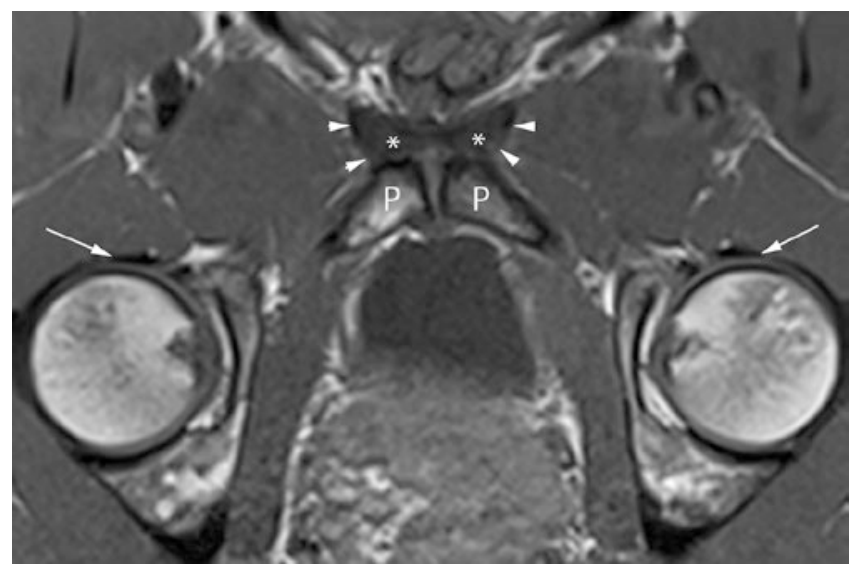

Fig. 6.2 Normal anatomy, MR imaging. Axial oblique T1 MR image shows pubic bodies $(\mathrm{P})$ and the adductor group tendons (arrowheads) beginning to separate from the aponeurotic tissues (*). Normal iliopsoas tendons (small arrows). (Reproduced with permission from reference Madani H, Robinson P. Top-Ten Tips for Imaging Groin Injury in Athletes. Semin Musculoskelet Radiol. 2019;23(4):361-75.)

Although it can be difficult to pinpoint a precise pain generator clinically, patients often present with orthopedic complaints that are most severe in a particular region of the pelvis: anterior, lateral, inferior, or posterior. (Note: The hip joint is covered in a separate chapter.) In this chapter, we highlight the most important injury patterns in each of these four locations, with a special emphasis on the anterior pelvis. abdominis tendon (white arrow). Pyramidalis present anteriorly (asterisk). (c) Lateral to (a) pubic marrow and cortex (P) with thin layer of intermediate signal hyaline cartilage (small arrowheads) closely applied to the anterior capsular tissues (between large white arrowheads). Merging with this tissue are the rectus abdominis tendon (RAb), pyramidalis (asterisk), superficial adductor longus tendon (arrows), deeper muscle (AL), and adductor brevis ( $\mathrm{ABr}$ ) muscle. (Reproduced with permission from Robinson P, Salehi F, Grainger A, et al. Cadaveric and MRI Study of the Musculotendinous Contributions to the Capsule of the Symphysis Pubis. American Journal of Roentgenology. 2007;188(5):W440-W445.)

\subsection{Anterior Pelvis}

\subsubsection{Who Is Most Likely to Get Imaging for Anterior Pain?}

At the anterior aspect of the pelvis, orthopedic imaging is often performed in athletes with pain. Athletic-related groin pain can occur with acute injuries, but commonly presents with an insidious onset owing to overuse. Sports that involve intense pivoting, twisting, and kicking are often implicated (e.g., soccer/football, rugby, hockey).

- In professional ice hockey players, for example, over half of all players experience groin and hip problems in a typical season, with almost one-third losing time from playing their sport [5].

- Other athletes also can experience groin pain, including dancers, swimmers, runners, and even unicyclists. In a recent study of 1304 unicyclists (who actively use adductor and other muscles), 17\% complained of groin pain [6]!

\subsubsection{What Is the Optimal Imaging Workup?}

Radiography is the initial screening test of choice in many practices. Unfortunately, radiography is often insensitive and non-specific in patients with chronic groin pain. 

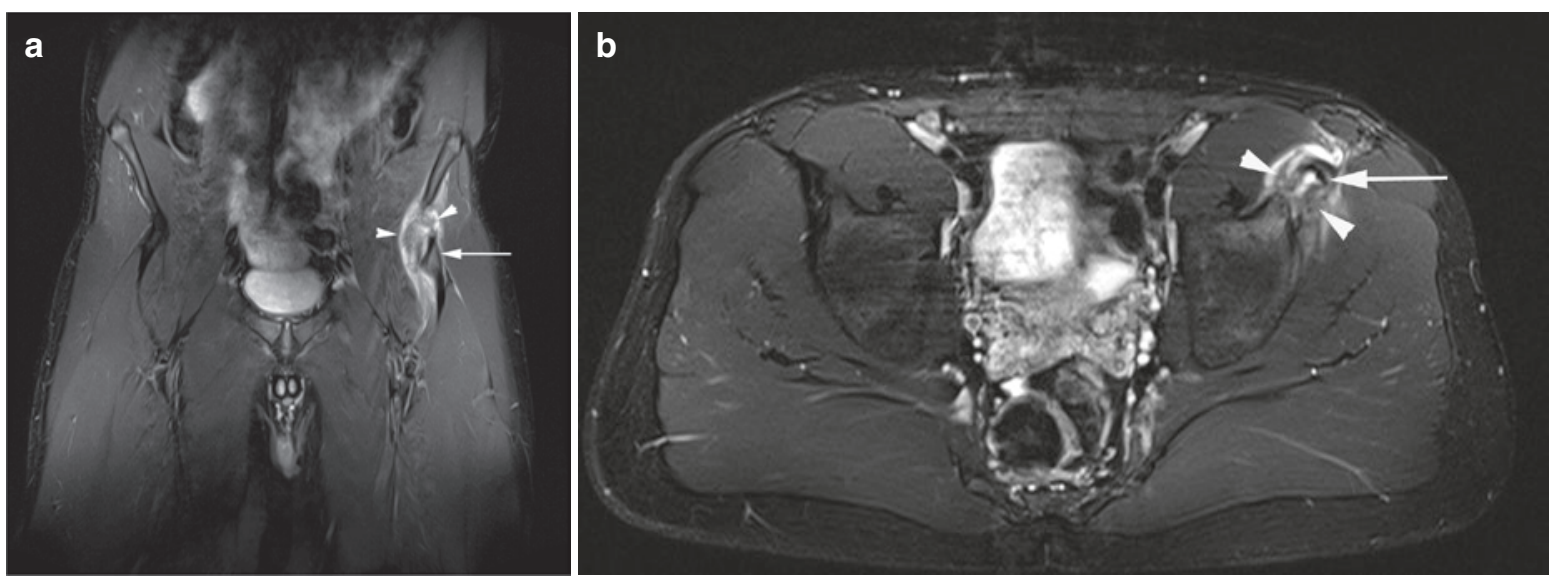

Fig. 6.3 Apophyseal injury. A 16-year-old rugby player with sudden anterior hip pain. (a) Coronal and (b) axial T2FS MR images show avulsion of the left anterior inferior iliac apophysis (arrow), hematoma (arrowheads), and intact indirect head of rectus femoris
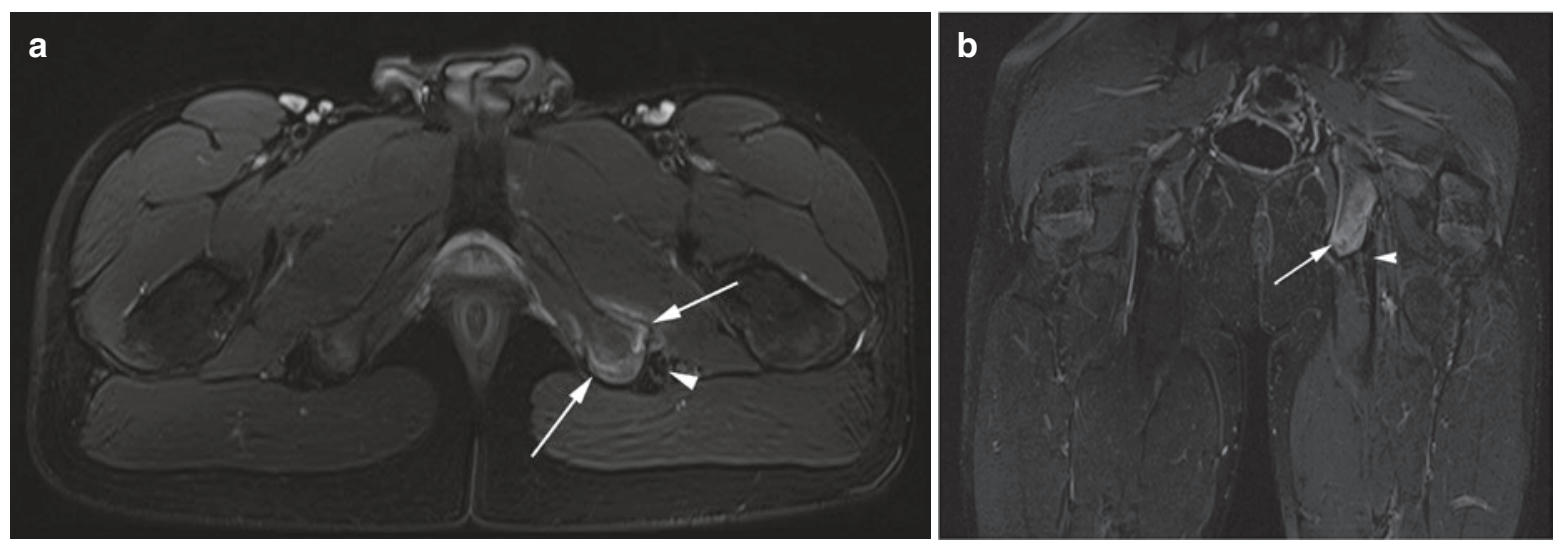

Fig. 6.4 Apophyseal injury. A 17-year-old soccer player with subacute hamstring pain. (a) Axial and (b) coronal T2FS MR images show left ischial apophysis edema, stress fracture and periosteal reaction (arrow), and normal hamstring tendons (arrowheads)
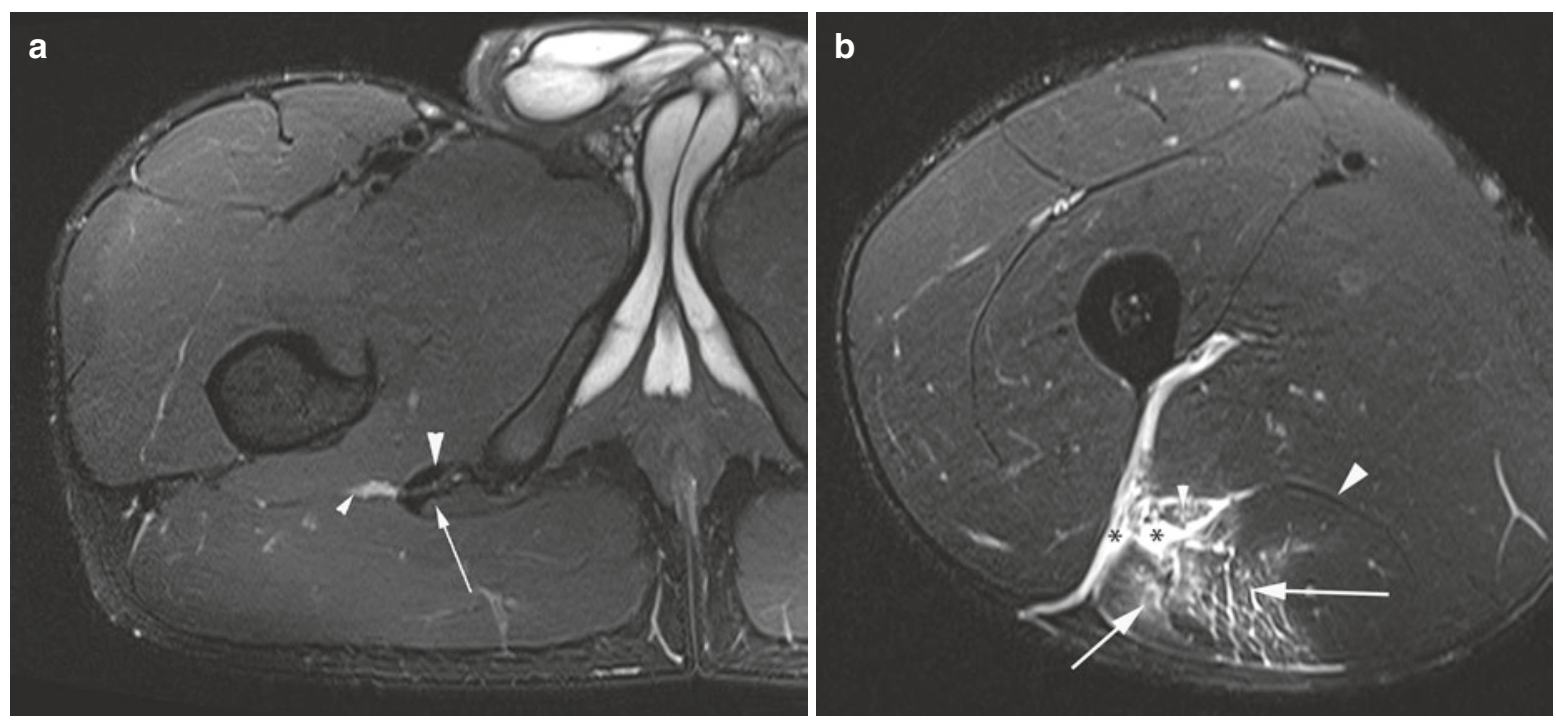

Fig. 6.5 Myotendinous injury. A 24-year-old soccer player with acute hamstring pain. Axial T2FS MR images show (a) right proximal thigh biceps femoris myotendinous tear with tendon involvement (arrows) with decompressed hematoma (asterisk) around the sciatic nerve (small with normal conjoint tendon (arrow), semimembranosus (large arrowhead), and sciatic nerve (small arrowhead) and (b) semitendinosus and arrowhead) and fascia. Normal semimembranosus (large arrowhead) 

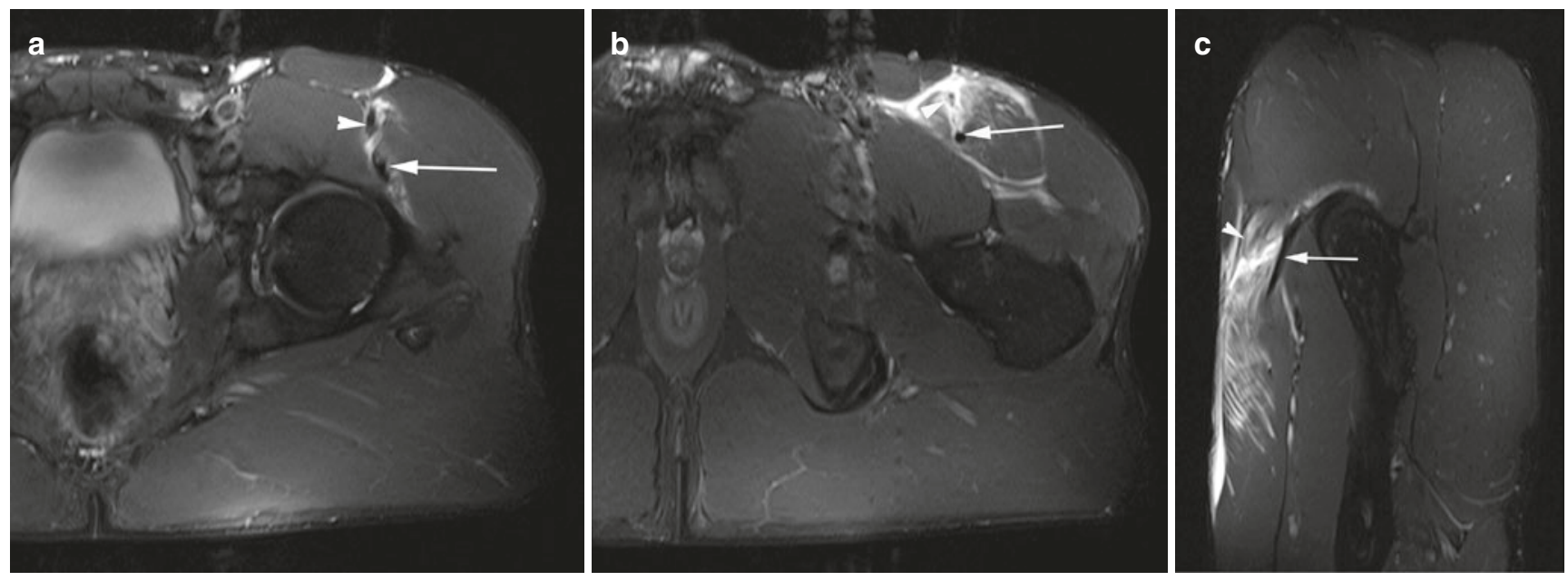

Fig. 6.6 Myotendinous injury. A 23-year-old soccer player with acute anterior hip pain. Axial and sagittal T2FS MR images show (a) left proximal thigh intact indirect head tendon (arrow), direct head tendon (arrowhead), and muscle edema. (b) and (c) Inferior to (a) show intact indirect head tendon (arrow), torn direct head tendon (arrowhead), and extensive muscle disruption
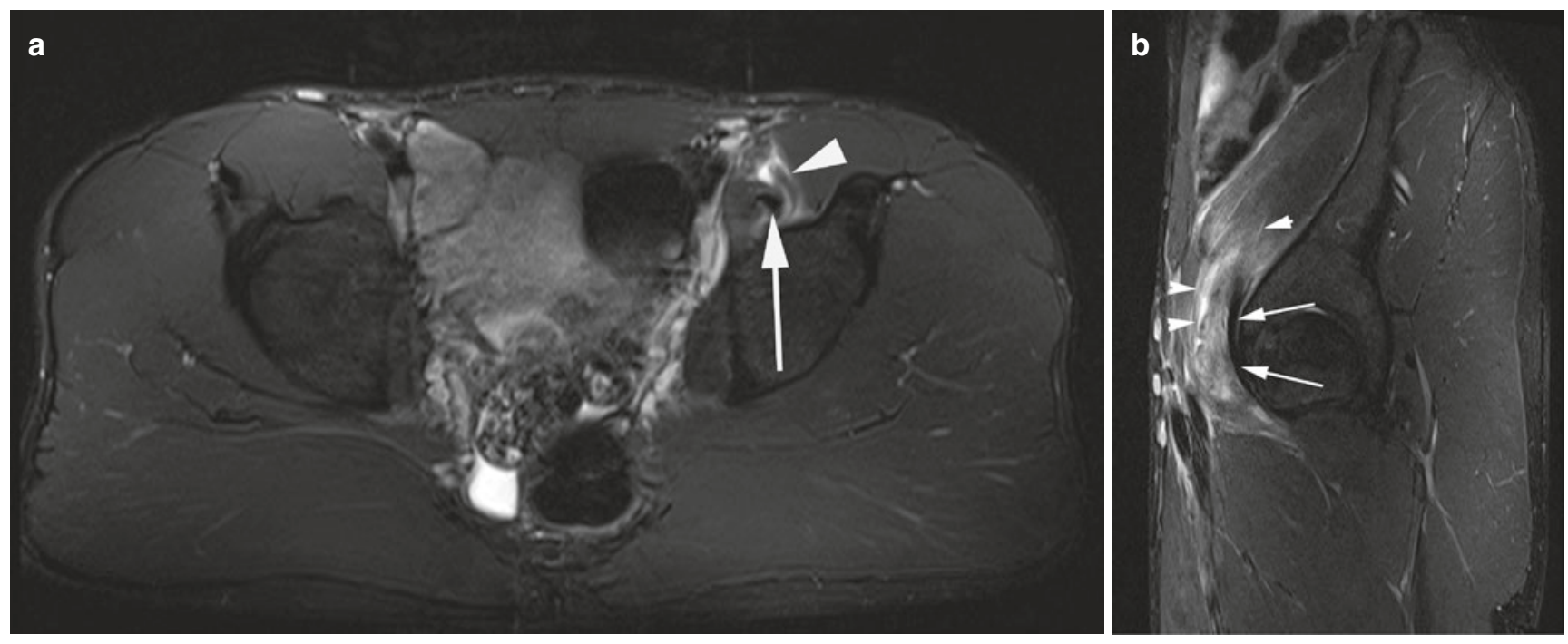

Fig. 6.7 Myotendinous injury. A 21-year-old soccer player with acute anterior hip pain. (a) Axial and (b) sagittal T2FS MR images show intact psoas major tendon (arrow) and myotendinous iliacus injury (arrowhead)

Indeed, sonography and MRI have become frontline tools in many practices owing to the low diagnostic yield of radiography, as well as concerns related to radiation exposure associated with radiography, CT, and scintigraphy in young athletes.

Sonography has numerous strengths, including point-ofcare evaluation, dynamic assessment (e.g., for hernia [7], tendon snapping), real-time sonopalpation at the exact site of pain, and guiding percutaneous injections for diagnostic and therapeutic purposes $[8,9]$.

- Sonography is best performed with a systematic protocol $[10,11]$. Using a high-frequency (e.g., $\geq 14 \mathrm{MHz}$ ) linear array transducer, the patient is positioned supine with the hips abducted and externally rotated. First, imaging is performed of the rectus abdominis/adductor aponeurosis (in the transverse and longitudinal planes) and the adductor longus attachment (in an oblique sagittal plane longitudinal to these fibers and in an orthogonal transverse plane). Then, dynamic imaging during Valsalva can be used assessing for an inguinal or femoral hernia [12], as well as the controversial finding of inguinal wall bulging believed by some to cause ilioinguinal nerve irritation. If indicated, additional evaluation of the iliopsoas and hip region also can be performed (e.g., for tendon snapping, iliopsoas bursitis, hip effusion). 
$M R I$ is better able to show soft tissue edema than sonography, as well as a wide spectrum of abnormalities within bones.

- MRI protocols commonly combine both a small and large field of view (FOV). The high spatial resolution images with a small FOV (e.g., 16-25 cm) have thin slices (e.g., 3 $\mathrm{mm}$ ) and are generally performed in the sagittal plane and an oblique-axial plane (paralleling the anterior tilt of the pelvic brim). Additional images with a large FOV (e.g., $38-40 \mathrm{~cm}$ ) have thicker slices (e.g., 4-5 mm) covering the pelvis, which allows screening of the hips, sacroiliac joints, spine, and pelvic viscera. This is particularly helpful because symptoms may radiate from another site or may not be localized [13]. No gadolinium is needed.

\subsubsection{What Terminology Should Be Used?}

Terminology has evolved over time. A multispecialty expert group consensus statement suggests the use of an umbrella term "groin pain in athletes" (a general description that cannot be misunderstood as a diagnostic term), rather than previously used terms with variable definitions (e.g., athletic pubalgia, sports hernia, sportsman's groin) [14]. Although this entity may be caused by a wide variety of derangements at the hip or elsewhere, groin pain in athletes is often classified into four categories related to location: pubic, adductor, inguinal, and iliopsoas [14]. Pubic- and adductor-related groin pain have overlapping features and are discussed together here.

\subsubsection{Pubic and Adductor Groin Pain}

Anatomy. The pubic symphysis is a cartilaginous joint supported by four capsular ligaments (superior, inferior, anterior, posterior) and numerous musculotendinous attachments. Of all the abdominal and adductor muscles that converge in this region, the most important stabilizers are the rectus abdominis and the adductor longus. The adductor longus can be identified as the most anterior of the adductor muscle group.

Injury Patterns. Acutely, muscle tears (partial or complete) or avulsion injuries can occur near the pubic symphysis. Such injuries frequently target the adductor longus (Fig. 6.8) [15-17].

In the non-acute setting, the most common areas of injury are the rectus abdominis/adductor aponeurosis and pubic symphysis capsular attachment site. Athletes are predisposed to injury when shear stresses are accentuated by muscle imbalances (typically stronger adductor muscles with anteroinferior tension versus weaker abdominal muscles with superior tension). This allows initial low-grade overuse injuries to propagate into overt tears at the musculoaponeurotic attachments.

Imaging Findings. Although pubic bone marrow edema (BME) may be seen in asymptomatic athletes owing to chronic loading, more severe and extensive BME is positively associated with pain. Pubic BME may be an isolated finding related to stress reaction, but it is crucial to look for additional abnormalities (Fig. 6.9). Three abnormalities associated with pubic BME are especially important to report: (1) stress fractures (typically with intense BME adjacent to a fracture line of low signal intensity), (2) apophysitis

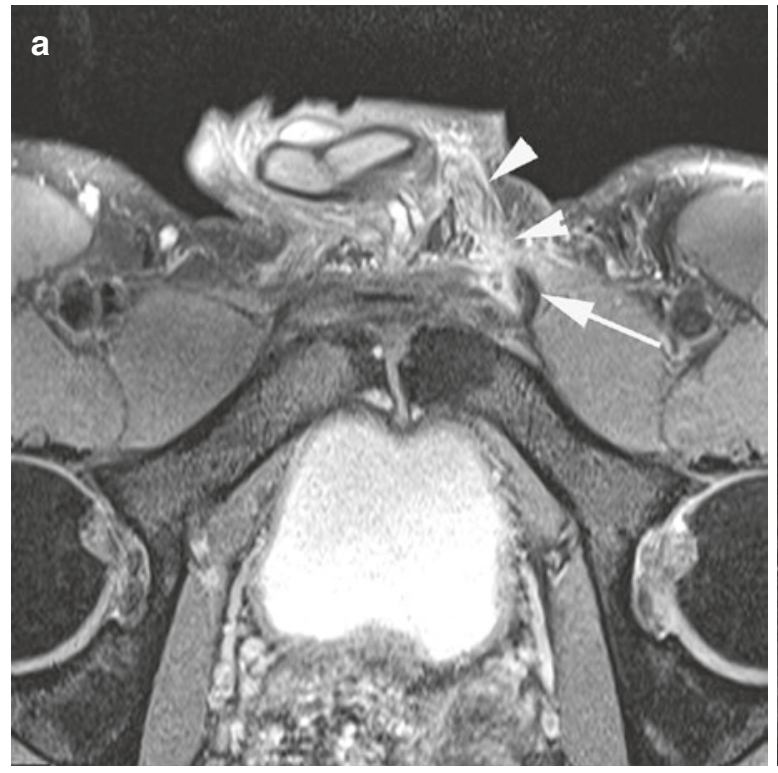

Fig.6.8 Acute groin injury. A 26-year-old soccer player with acute left groin pain. (a) Oblique axial PDFS and (b) coronal T2FS MR images show left adductor longus and pectineus tendon avulsion (arrow) with

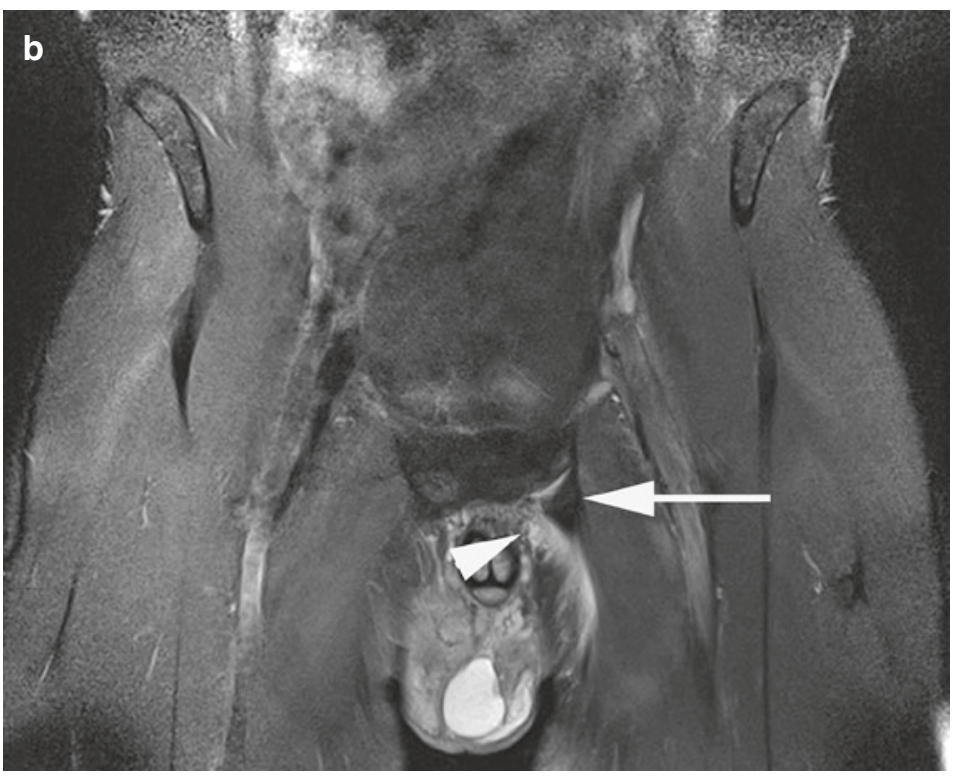

soft tissue hemorrhage extending into the left inguinal canal due to acutely torn aponeurosis (arrowheads) 

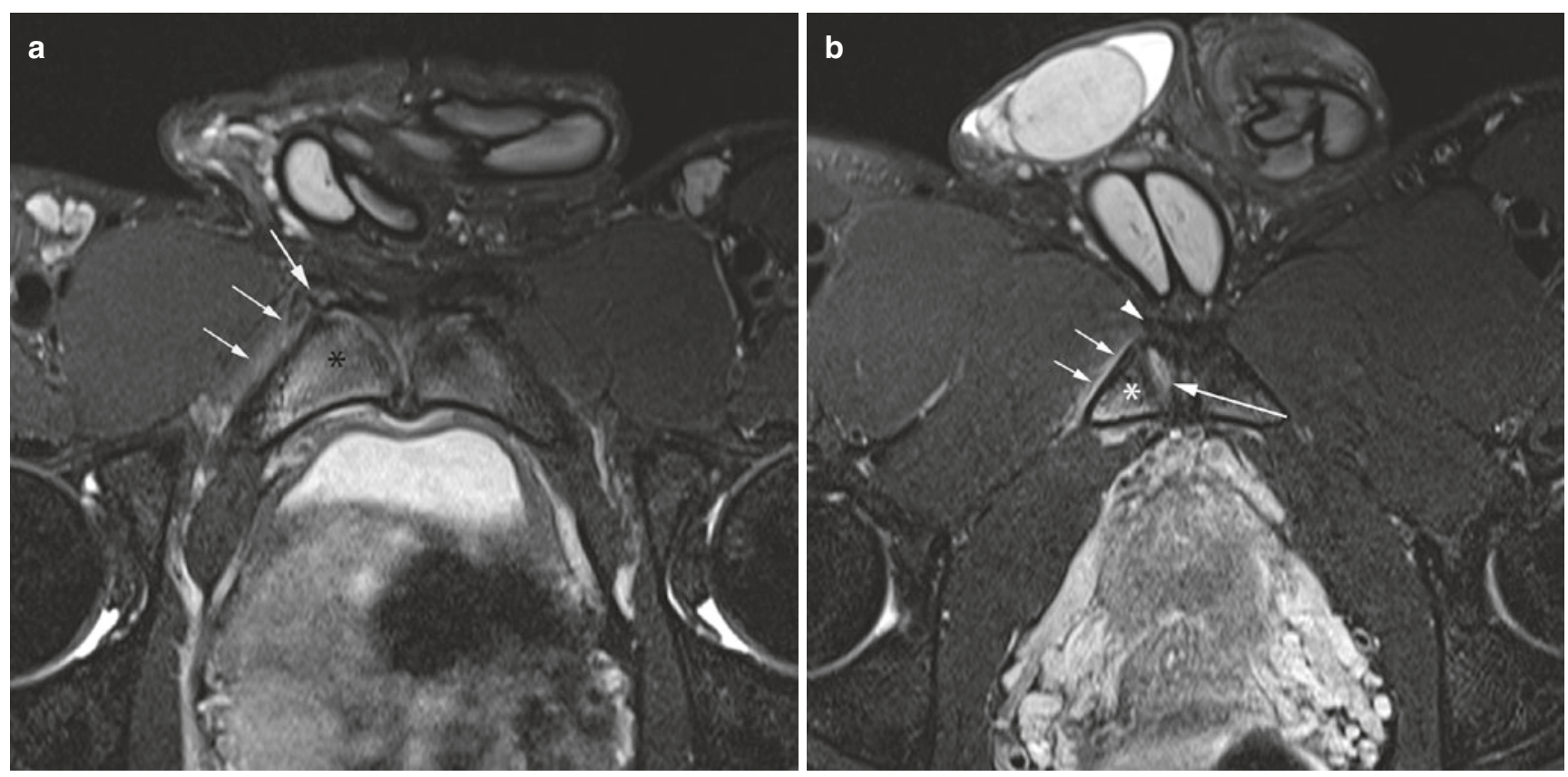

Fig.6.9 Unilateral edema pubic capsule aponeurotic and bone marrow. A 22-year-old professional soccer player with right-sided symptoms. (a) and (b) Sequential axial oblique PDFS MR images show right-sided high signal with no definite tear (large white arrow) between the pubis and main aponeurotic tissue. There is also mild soft tissue edema adja-

(i.e., T2 hyperintensity at the pubic apophysis, anteromedially in young athletes) (Fig. 6.10), and (3) rectus abdominis/ adductor aponeurosis injury (Fig. 6.11).

Rectus abdominis/adductor aponeurosis injury is among the most common imaging finding in athletes with chronic groin pain. Key MRI findings include edema or disruption at the aponeurosis or the capsular attachment. Such abnormalities go by variable names in the medical literature (e.g., "secondary cleft sign," "plate injury," "pubic aponeurotic defect," "overuse enthesitis"), and therefore objective reporting of anatomic location, signal intensity (e.g., mild to severe edema), and size is often recommended.

The most widely known MRI finding is the "secondary cleft sign" seen as fluid signal undermining the rectus abdominis/adductor aponeurosis attachment to the pubis (in communication with the pubic symphysis). Similar to BME, these MRI findings tend to be mild or absent in asymptomatic athletes, while more severe and extensive findings are positively associated with active injury. Tendinopathy in the adductors can also be sought, but imaging diagnosis of adductor tendinopathy has not been validated as highly reliable [18].

\subsubsection{Inguinal Groin Pain}

Anatomy. The inguinal canal is located superolateral to the pubic symphysis (above the medial half of the inguinal ligament) and is approximately $4 \mathrm{~cm}$ long. This tunnel transmits cent to the pubic cortex (small arrows). The left side shows no tear and minimal edema. There is diffuse moderate bilateral bone marrow edema $(*)$ more extensive on the right. (Reproduced with permission from reference Madani H, Robinson P. Top-Ten Tips for Imaging Groin Injury in Athletes. Semin Musculoskelet Radiol. 2019;23(4):361-75.)

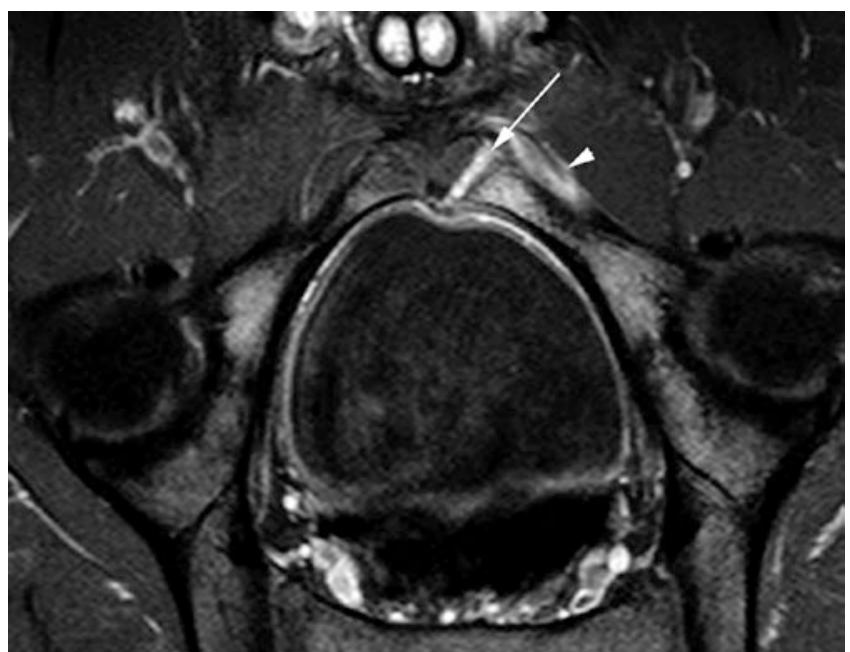

Fig.6.10 Apophyseal injury. A 16-year-old soccer player with severe left-sided pain. Axial oblique T2FS MR image shows severe left pubic body and ramus bone marrow edema with adjacent soft tissue edema (arrowhead). The apophyseal junction is ill-defined, widened, and of high signal (arrow). (Reproduced with permission from reference Madani H, Robinson P. Top-Ten Tips for Imaging Groin Injury in Athletes. Semin Musculoskelet Radiol. 2019;23(4):361-75.)

the spermatic cord in men or the round ligament in women, as well as associated neurovascular structures.

The inguinal aponeurotic falx or "conjoint tendon" (the aponeurosis of both the internal oblique and transverse muscles of the abdomen) merges with the sheath of the rectus abdominis muscle at a variable distance from the symphysis 

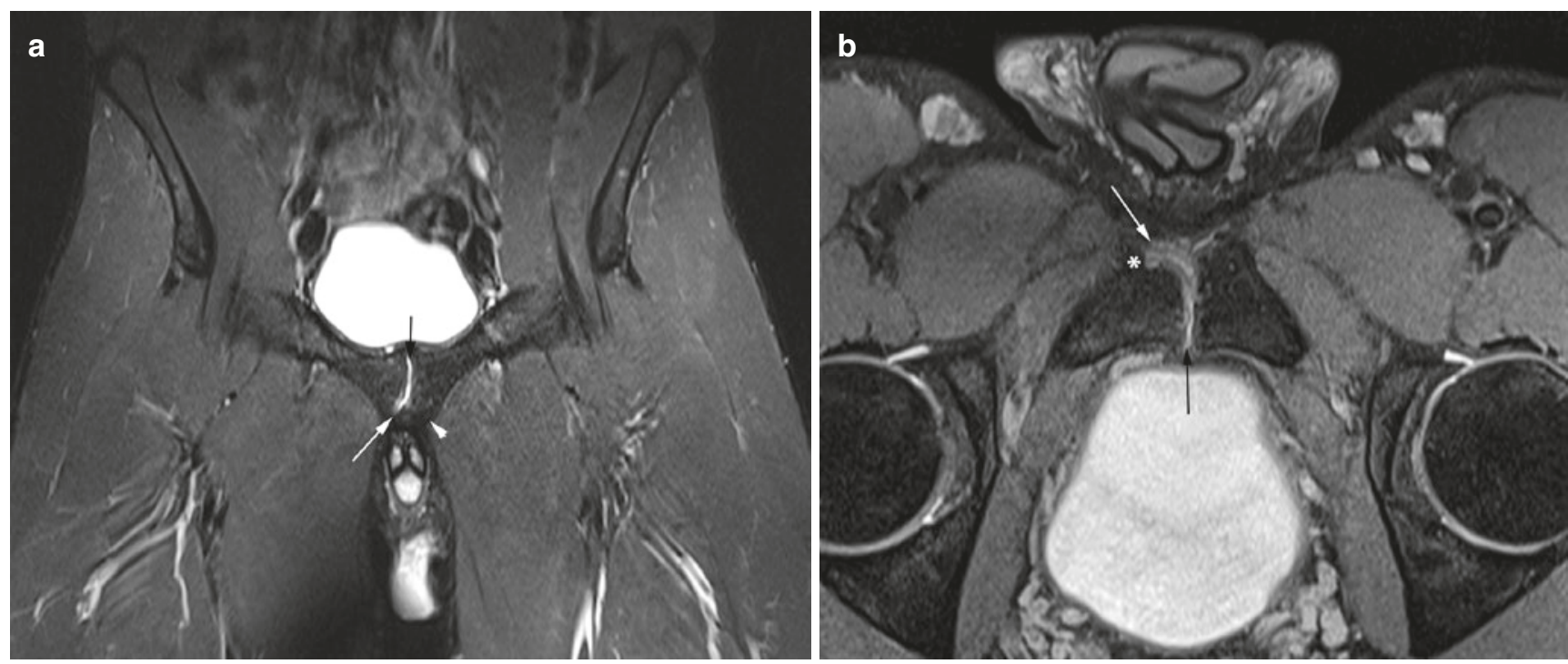

Fig. 6.11 Partial tear pubic capsule aponeurotic junction ("inferior cleft"). A 23-year-old professional rugby player with right-sided symptoms. (a) Coronal T2FS and (b) axial oblique PDFS images show normal joint (black arrow) and left soft tissue junction (white arrowhead) with right high signal tear (white arrow) between the pubis and main

tubercle. Recent research suggests that a high insertion of the conjoint tendon seen on MRI is associated with inguinalrelated groin pain in athletes [19].

Injury Patterns. Pain with tenderness at the inguinal canal region aggravated by a Valsalva-type maneuver is widely reported in the medical literature. However, in the young athletic population, no true hernia is typically palpable at clinical examination or visible at imaging. When a true hernia is present in this region, it is classically described as "indirect" (i.e., abdominal contents exit through the inguinal canal) or "direct" (i.e., exists through the abdominal wall deep layers, thereby bypassing the inguinal canal).

Imaging Findings. Imaging evaluation for true inguinal and femoral hernias is best performed by sonography. For patients with inguinal-related pain but not true hernia, the posterior inguinal wall can be evaluated for laxity (incompetence) that may possibly irritate the ilioinguinal nerve. Unfortunately, these findings are regarded as subjective and have disappointing diagnostic reliability. MRI evaluation in these patients is generally normal.

\subsubsection{Iliopsoas Groin Pain}

Anatomy. The iliopsoas is formed by the confluence of the iliacus muscle (at the inner aspect of the iliac fossa) and the psoas muscle. The tendon slips from the two muscle bellies may or may not fuse together prior to insertion onto the lesser trochanter; a normal longitudinal division between these two components should not be misdiagnosed as a longitudinal tendon tear. aponeurotic tissue. No bone marrow edema is evident though there is chronic remodeling $(*)$ present. (Reproduced with permission from reference Madani H, Robinson P. Top-Ten Tips for Imaging Groin Injury in Athletes. Semin Musculoskelet Radiol. 2019;23(4):361-75.)

The iliopsoas bursa is located between the iliopsoas and the hip joint. This is the largest bursa in the human body and communicates with the hip joint normally in up to approximately $15 \%$ of adults.

Injury Patterns. The iliopsoas is considered the least common source of athletic groin pain. Injuries are more common with repetitive microtrauma than with a single traumatic event. Typical maneuvers causing pain include getting up from sitting or squatting, as well as sometimes resisted hip flexion.

Imaging Findings. Sonography can be useful in the evaluation of snapping (e.g., dynamic assessment as the iliopsoas passing over the iliopectineal eminence), iliopsoas bursitis (i.e., hypoechoic fluid along the iliopsoas tendon), and guiding percutaneous injections.

MRI provides for simultaneous assessment of iliopsoas edema, iliopsoas bursitis, and derangements in adjacent structures like the hip (Fig. 6.12). Peritendinous edema associated with the iliopsoas bursa can be seen in the setting of overuse or strain injuries, as well as in some cases of snapping hip ("coxa saltans interna").

\section{Key Point}

- The differential diagnosis of groin pain in athletes is broad and includes pubic, adductor, inguinal, and iliopsoas etiologies. Knowledge of the anatomy in each region allows for optimized diagnosis with sonography and MRI. 


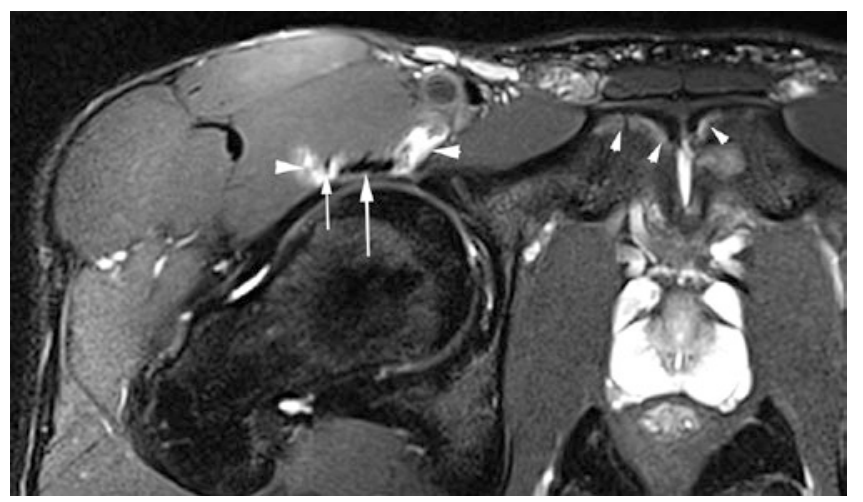

Fig. 6.12 Iliopsoas bursitis. A 23-year-old soccer player with rightsided pain. Axial T2FS MR image shows marked edema and bursitis (arrowheads) surrounding both the psoas (large arrow) and iliacus (small arrow) components of the iliopsoas tendon anterior to a normal hip joint. Note the normal pubic apophyses (small white arrowheads). (Reproduced with permission from reference Madani H, Robinson P. Top-Ten Tips for Imaging Groin Injury in Athletes. Semin Musculoskelet Radiol. 2019;23(4):361-75.)

\subsection{Lateral Pelvis}

Anatomy. The abductor tendons evaluated most commonly are the gluteus minimus and gluteus medius. The gluteus minimus inserts predominantly onto the anterior facet of the greater trochanter, with some fibers also merging with the hip joint capsule. The gluteus medius has two insertion sites on the greater trochanter, with a thicker portion inserting at the superoposterior facet and a thinner portion inserting distally at the lateral facet [20]. Together, these two gluteal tendons are often referred to as "the abductor tendons" or "rotator cuff of the hip." Several bursae adjacent to the greater trochanter have been described, with the largest located superficial to the posterior facet (the trochanteric bursa).

At the lateral aspect of the pelvis, the iliotibial band (ITB) (anteriorly) and gluteal aponeurotic fascia (posteriorly) originate at the margin of the iliac crest, descending superficial to the gluteus medius and greater trochanter [21].

Injury Patterns. Trochanteric pain syndrome is generally caused by abnormalities involving the abductor tendons. Although peritrochanteric edema and bursal fluid are commonly present with trochanteric pain syndrome, they are generally not reliably associated with symptoms [22].

The ITB and gluteal aponeurotic fascia can be injured with trauma or repetitive microtrauma. In addition to apophyseal derangements in skeletally immature patients and enthesitis at the osseous attachment in adults, the proximal ITB is a characteristic site of overuse injuries (e.g., in runners).

Imaging Findings. Tendinopathy and tearing in the abductor tendons become increasingly prevalent with advanc-

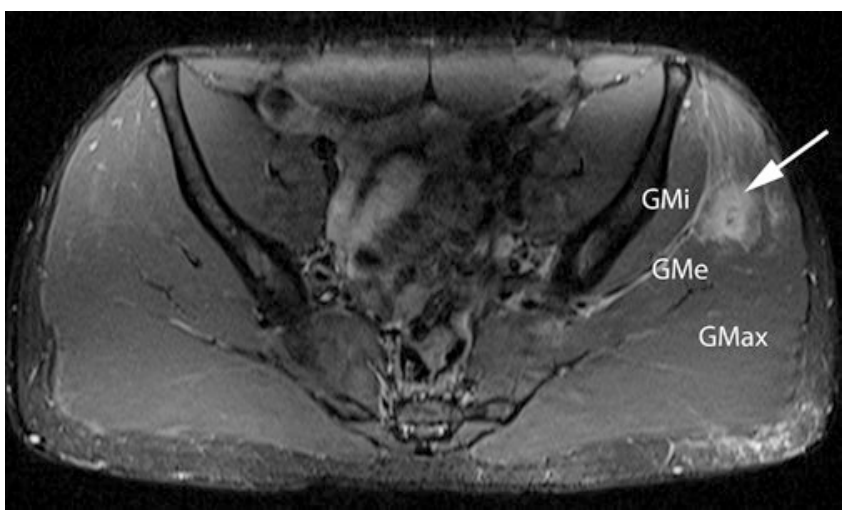

Fig. 6.13 Myotendinous injury. A 23-year-old soccer player with acute buttock pain. Axial T2FS MR image shows left gluteus medius myotendinous tear (arrow). The distal tendon was normal and intact

ing age. As with rotator cuff tears in the shoulder, these tendon tears typically are located near the osseous insertion site and begin as partial-thickness tears (fiber discontinuity and T2 hyperintensity). Incomplete abductor tendon tears are most commonly reported at the distal-anterior fibers of the gluteus medius tendon [20]. Some tears are discovered as incidental, non-acute findings. Chronic tears associated with muscle atrophy and fatty infiltration have suboptimal surgical outcomes. Acute tears in the muscle belly of athletes are relatively uncommon but can occur (Fig. 6.13).

"Proximal ITB syndrome" typically occurs superolateral to the hip joint and may be overlooked at the margin of the MRI FOV. Characteristic findings include edema along the proximal ITB, often with subtle fiber thickening at the proximal attachment or a small area of ill-defined partial tearing.

\section{Key Point}

- At the lateral aspect of the pelvis, derangements may occur in the abductor tendons, ITB, and gluteal aponeurotic fascia. Partial abductor tears are most commonly reported at the distal-anterior gluteus medius tendon. Disorders at the ITB and gluteal aponeurotic fascia regions include apophysitis, enthesitis, and proximal iliotibial band syndrome.

\subsection{Inferior Pelvis}

Anatomy. The hamstring tendons originate at the ischial tuberosity, with the semimembranosus tendon anteriorly and the conjoined tendon of the biceps femoris (long head) and semitendinosus posteriorly. (If you have difficulty remembering that it's the biceps femoris and semitendinosus that form the conjoined tendon, just remember that "best friends stick together"!) 
Ischiofemoral impingement is associated with a narrowed space between the ischial tuberosity and the femur. Predisposing factors may be developmental (e.g., pelvic morphology resulting in increased ischial and femoral neck angles [23], femoral morphology resulting in increased femoral version or coxa valga [24]) or acquired (e.g., heterotopic ossification, post-traumatic deformity).

Injury Patterns and Imaging Findings. In the acute setting, hamstring muscle eccentric contraction can result in strain-type injuries that usually involve the myotendinous junction and are treated non-operatively in young adult athletes (as shown in Fig. 6.5, above). Tendon ruptures at the proximal attachment are much less common in young adults but are important to recognize because they may be repaired surgically (particularly if at least two-thirds of the tendon fibers are ruptured and retracted) (Fig. 6.14).

In the chronic setting, hamstring tendon tears are associated with scarring that may tether the sciatic nerve and make delayed repair more difficult. In older adults, hamstring tendinopathy and attritional partial tears are extremely common (e.g., interstitial tearing or stripping at the conjoined tendon attachment). Hamstring tendinopathy with symptomatic peritendinous edema in the adjacent bone and soft tissues also can occur in young adult athletes owing to overuse (Fig. 6.15).

Ischiofemoral impingement on MRI is defined by narrowing of the ischiofemoral space $(\leq 15 \mathrm{~mm})$ or quadratus
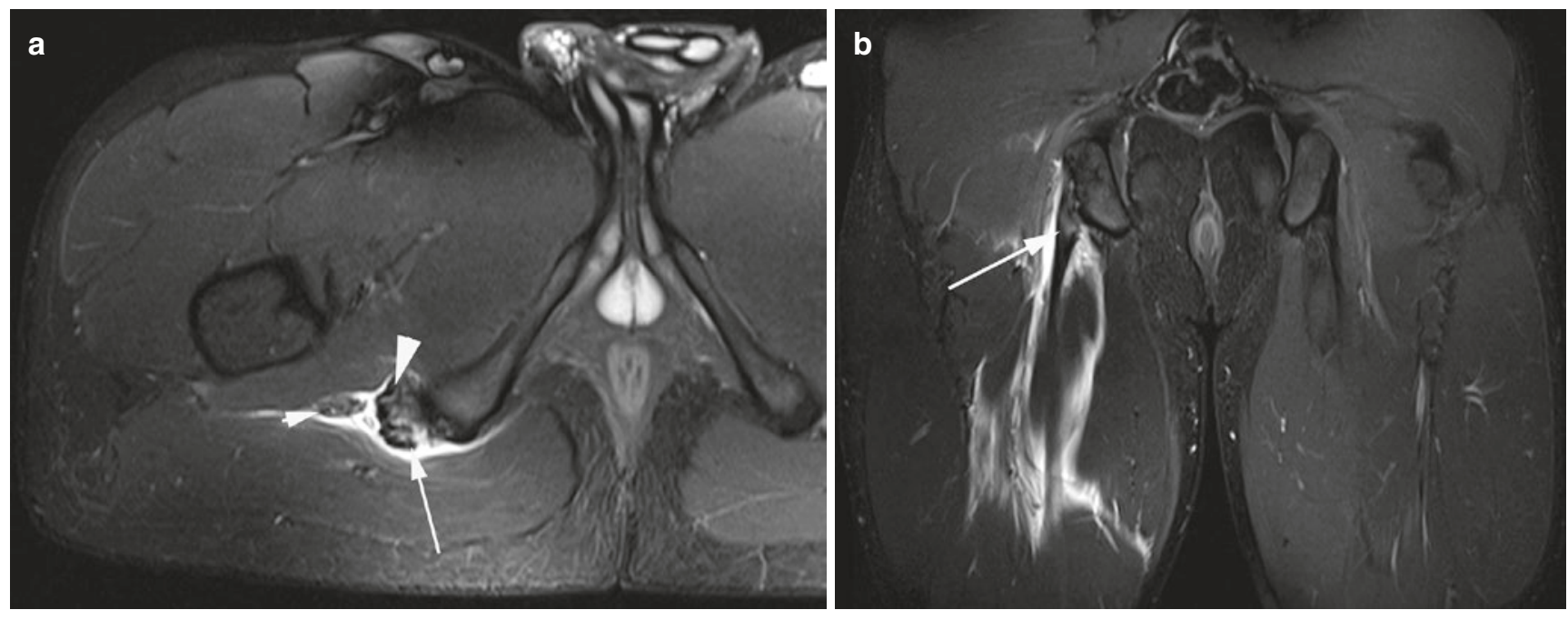

Fig. 6.14 Tendon injury. A 26-year-old soccer player with acute hamstring pain. (a) Axial and (b) coronal T2FS MR images show right con-

proximal semimembranosus tendon (large arrowhead). Adjacent sciatic joint tendon origin partial tear (arrow), edematous but grossly intact
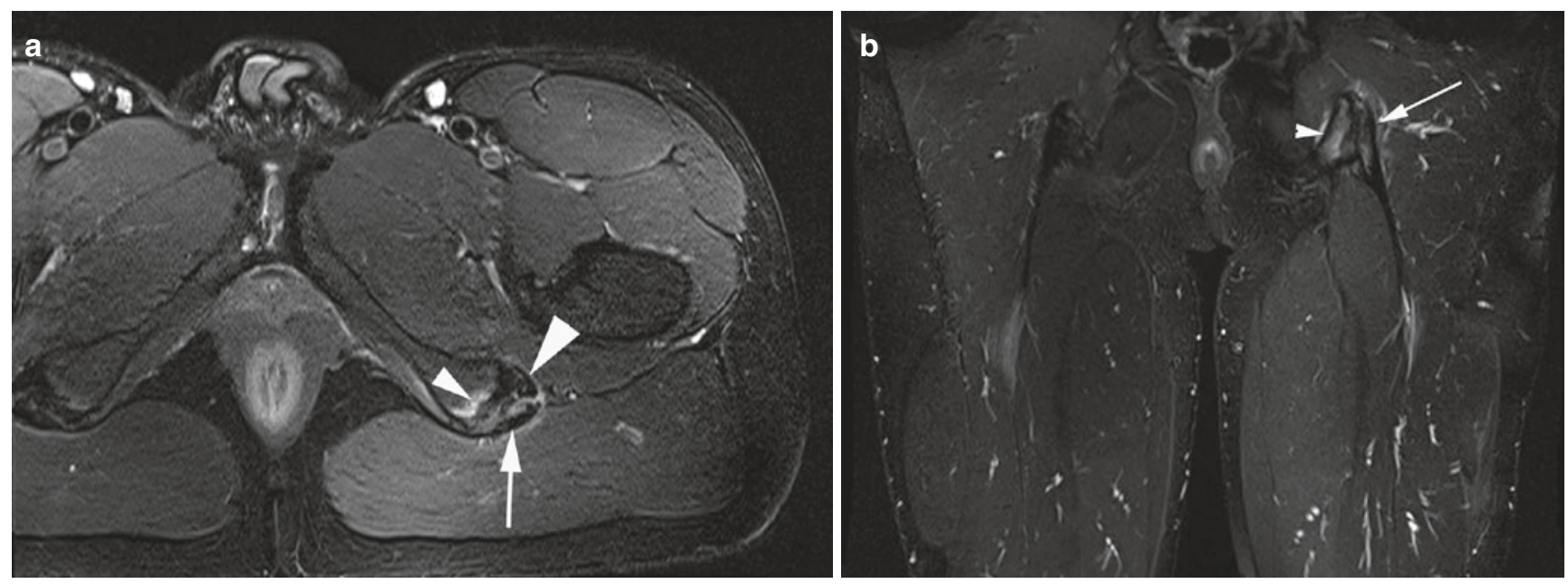

Fig. 6.15 Tendinopathy. A 25-year-old soccer player with subacute hamstring pain. (a) Axial and (b) coronal T2FS MR images show left ischial subcortical bone marrow edema (small arrowhead), paratenon edema, intrinsic conjoint tendon oedema (arrow), and normal semimembranosus tendon (large arrowhead) 
femoris space $(\leq 10 \mathrm{~mm})$ with associated quadratus femoris edema or fatty infiltration/atrophy (Fig. 6.16). The ischiofemoral interval, however, varies with patient positioning. With active external rotation during kinematic MRI, for example, dynamic narrowing of the space occurs [25]. Interestingly, compared with matched controls, patients with ischiofemoral impingement have a higher prevalence of abductor tears and abductor muscle atrophy, suggesting to some investigators that abductor tears might be causally associated with ischiofemoral impingement [26].

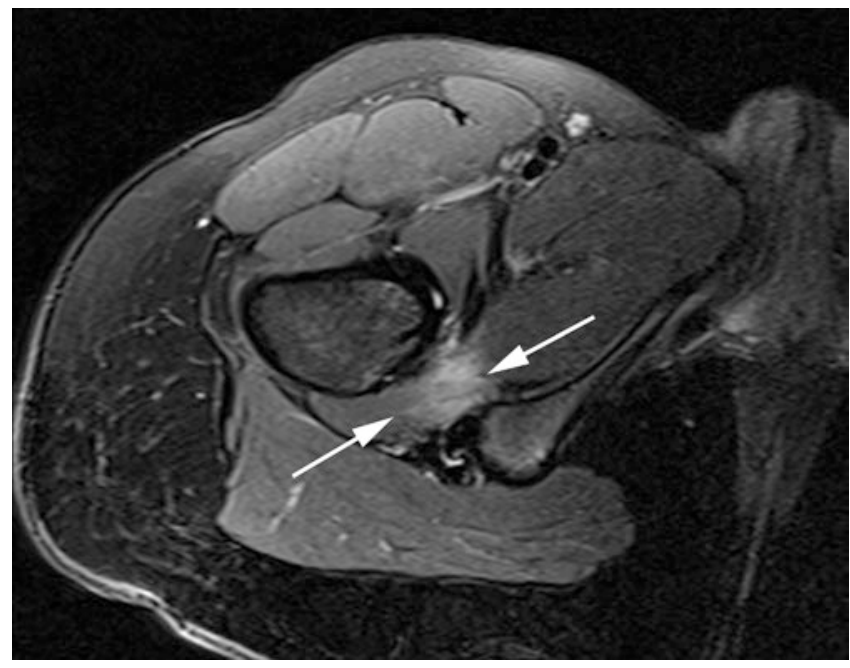

Fig. 6.16 Ischiofemoral impingement. A 22-year-old female recreational athlete with chronic hip pain. Axial PDFS MR image shows diffuse edema in the quadratus femoris muscle (arrows) bridging between the ischium and lesser trochanter

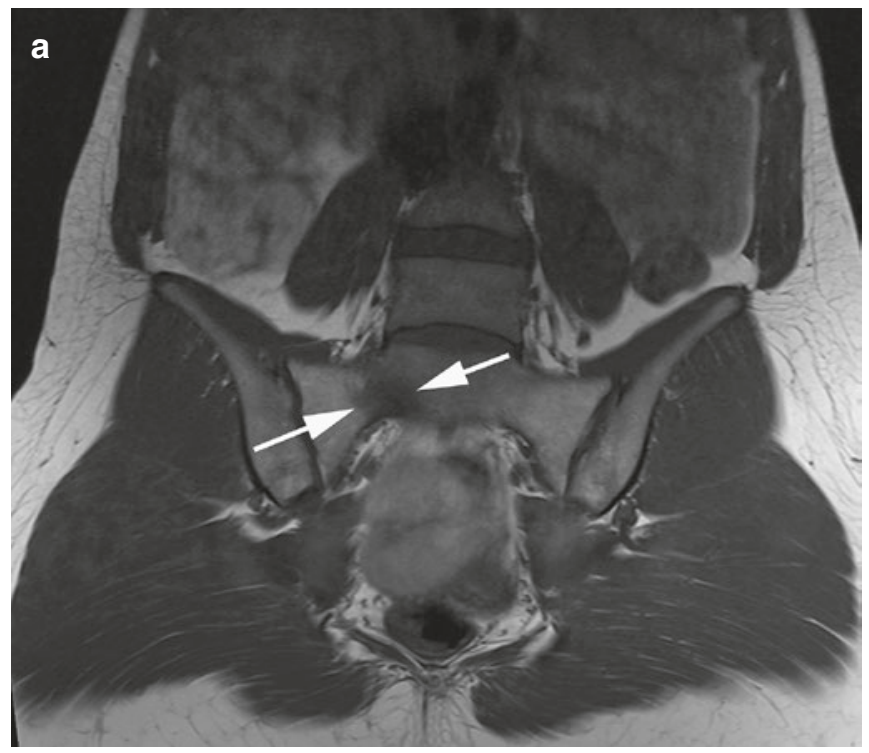

\section{Key Point}

- At the inferior aspect of the pelvis, hamstring disorders and ischiofemoral impingement are well characterized by MRI.

\subsection{Posterior Pelvis}

Anatomy. The sacrum typically has five fused segments in adults, with four pairs of anterior and posterior foramina, as well as a normal opening ("sacral hiatus") posteriorly at the S4/S5 level [27].

The coccyx has four segments in $\sim 75 \%$ of the population, but the number of segments varies from three to five. The intercoccygeal joints may or may not be fused and have highly variable alignment [27].

Injury Patterns and Imaging Findings. Both traumatic fractures and stress fractures occur in the sacrum (Fig. 6.17). These fractures may be described as involving the ala (zone I), the foraminal region (zone II), or the sacral spinal canal (zone III) [28]. In trauma patients, isolated zone I fractures are usually not associated with neurological deficits $(<10 \%)$ and are treated conservatively, while zone III fractures are more commonly associated with neurological deficits (> $50 \%$ ) and surgical treatment (especially for displaced or unstable fractures) [28].

With acute trauma to the sacrum or coccyx, it has been recommended that radiography be eliminated. Radiography misses most sacral fractures, with a sensitivity often cited at $30 \%$ [29]. Consequently, even in the setting of known or sus-

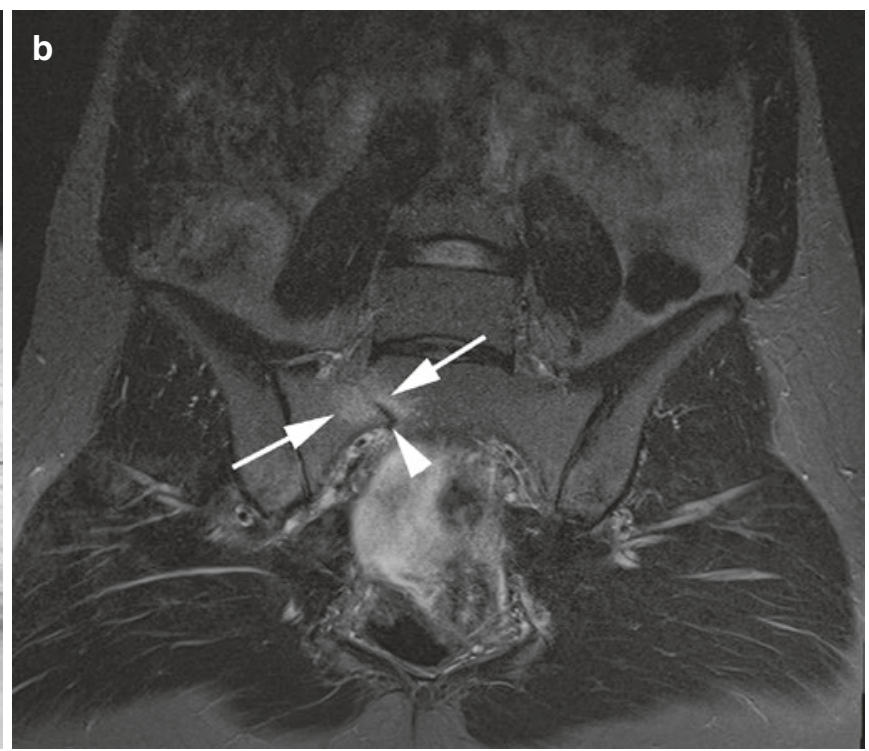

Fig. 6.17 Stress fracture. A 24-year-old male recreational athlete with subacute low back pain. Coronal (a) T1 and (b) T2FS MR images show right-sided focal sacral bone marrow edema (arrows) and fracture line (arrowhead) 
pected trauma, radiography is not considered the initial diagnostic imaging test of choice in many settings. In the emergency department setting, for example, it has been recommended that radiography of the sacrum and coccyx be eliminated because it can actually result in increased wait times, delayed follow-up imaging, and substantial radiation exposure [29]. Rather, patients may benefit from being treated conservatively or receiving advanced imaging based on clinical parameters.

In geriatric patients with bilateral fragility fractures of the sacrum [30], fractures start with unilateral sacral disruption, followed by contralateral sacral alar fracture and then an interconnecting transverse fracture in at least half of all cases (more commonly at S1/S2 than S2/S3).

With chronic coccyx pain (coccydynia), radiography is controversial. Advocates believe that lateral radiography in the seated position can reveal excessive motion (compared to the standing position) (defined as $>25 \%$ posterior subluxation or $>25$ degrees of flexion with sitting, compared to standing) [27]. Detractors of coccygeal radiography note the high pelvic radiation dose and the low diagnostic yield, including unreliable assessment for important abnormalities like fracture, neoplasm, and infection. On MRI, a small FOV technique in patients with coccydynia can show edema that is related to mechanical irritation and abnormal motion [27]. Depending on the etiology of coccydynia, the diverse menu of treatment options includes percutaneous injection (e.g., of the ganglion impar), cement augmentation (coccygeoplasty), extracorporeal shock wave therapy, neuromodulation of dorsal root ganglia, and coccygectomy.

\section{Key Point}

- At the posterior aspect of the pelvis, pain in the sacrum and coccyx is a common clinical complaint. Rather than routine radiography, patients may benefit from being treated conservatively or receiving advanced imaging based on clinical evaluation. In evaluation of small abnormalities, high spatial resolution cross-sectional imaging may help direct patient management.

\subsection{Concluding Remarks}

Pain in the groin and pelvis are common, but accurate clinical diagnosis can be challenging. Patients commonly localize orthopedic symptoms to the anterior, lateral, inferior, or posterior aspect of the pelvis. Each of these four locations has its own intricate anatomy, distinctive disorders, and treatment pathways. By optimizing our knowledge and imaging techniques in each location, we are able to add value by optimally "ruling in" and "ruling out" key diagnoses, thus optimizing patient management and outcomes.

\section{Take Home Messages}

- Although groin pain in athletes can originate at the hip or elsewhere, groin pain is often classified into four categories related to location: pubic, adductor, inguinal, and iliopsoas.

- At the anterior aspect of the pelvis, the most important areas of injury in athletes include the pubic symphysis capsular ligaments and the rectus abdominis/adductor aponeurosis attachment sites.

- At the lateral aspect of the pelvis, tendinopathy and tearing of the abductor tendons are common pain generators. Important injuries also may occur near the margin of the iliac crest, including proximal ITB syndrome.

- At the inferior aspect of the pelvis, hamstring disorders and ischiofemoral impingement are frequently evaluated by MRI.

- At the posterior aspect of the pelvis, derangements in the sacrum and coccyx are often missed with conventional radiography. Patients should be advanced to cross-sectional imaging in the appropriate clinical context.

\section{References}

1. Ricci PL, Maas S, Gerich T, Kelm J. Influence of pubic symphysis stiffness on pelvic load distribution during single leg stance. Int $\mathbf{J}$ Numer Methods Biomed Eng. 2020;36:e3319.

2. Meyers AB, Laor T, Zbojniewicz AM, Anton CG. MRI of radiographically occult ischial apophyseal avulsions. Pediatr Radiol. 2012;42:1357-63.

3. Sailly M, Whiteley R, Read JW, Giuffre B, Johnson A, Hölmich P. Pubic apophysitis: a previously undescribed clinical entity of groin pain in athletes. Br J Sports Med. 2015;49:828-34.

4. Serner A, Weir A, Tol JL, et al. Characteristics of acute groin injuries in the hip flexor muscles - a detailed MRI study in athletes. Scand J Med Sci Sports. 2018;28:677-85.

5. Wörner T, Thorborg K, Eek F. High prevalence of hip and groin problems in professional ice hockey players, regardless of playing position. Knee Surg Sports Traumatol Arthrosc. 2020;28:2302-8.

6. Kobayashi D, Kobayashi N, Oishi T, et al. Prevalence of groin pain in unicycle athletes: a nationwide questionnaire survey. J Orthop Surg (Hong Kong). 2020;28:2309499020934201.

7. Vasileff WK, Nekhline M, Kolowich PA, Talpos GB, Eyler WR, van Holsbeeck M. Inguinal hernia in athletes: role of dynamic ultrasound. Sports Health. 2017;9:414-21.

8. Brandon CJ, Jacobson JA, Fessell D, et al. Groin pain beyond the hip: how anatomy predisposes to injury as visualized by musculoskeletal ultrasound and MRI. Am J Roentgenol. 2011;197:1190-7. 
9. Yeap PM, Robinson P. Ultrasound diagnostic and therapeutic injections of the hip and groin. J Belg Soc Radiol. 2017;101(Suppl 2):6.

10. Madani H, Robinson P. Top-ten tips for imaging groin injury in athletes. Semin Musculoskelet Radiol. 2019;23:361-75.

11. Morley N, Grant T, Blount K, Omar I. Sonographic evaluation of athletic pubalgia. Skeletal Radiol. 2016;45:689-99.

12. Gupta H, Subedi N, Robinson P. Effectiveness of sonography in detecting clinically occult femoral hernias. J Ultrasound Med. 2016;35:1675-9.

13. Robinson P, Grainger AJ, Hensor EMA, Batt ME, O'Connor PJ. Do MRI and ultrasound of the anterior pelvis correlate with, or predict, young football players' clinical findings? A 4-year prospective study of elite academy soccer players. Br J Sports Med. 2015;49:176-82.

14. Weir A, Brukner P, Delahunt E, et al. Doha agreement meeting on terminology and definitions in groin pain in athletes. Br J Sports Med. 2015;49:768-74.

15. Serner A, Roemer FW, Hölmich P, et al. Reliability of MRI assessment of acute musculotendinous groin injuries in athletes. Eur Radiol. 2017;27:1486-95.

16. Serner A, Weir A, Tol JL, et al. Associations between initial clinical examination and imaging findings and return-to-sport in male athletes with acute adductor injuries: a prospective cohort study. Am J Sports Med. 2020;48:1151-9.

17. Schilders E, Mitchell AWM, Johnson R, Dimitrakopoulou A, Kartsonaki C, Lee JC. Proximal adductor avulsions are rarely isolated but usually involve injury to the PLAC and pectineus: descriptive MRI findings in 145 athletes. Knee Surg Sports Traumatol Arthrosc. 2020; https://doi.org/10.1007/s00167-020-06180-5. Epub ahead of print. PMID: 32767053

18. Branci S, Thorborg K, Nielsen MB, Hölmich P. Radiological findings in symphyseal and adductor-related groin pain in athletes: a critical review of the literature. Br J Sports Med. 2013;47:611-9.

19. Bou Antoun M, Ronot M, Crombe A, Moreau-Durieux MH, Reboul G, Pesquer L. High insertion of conjoint tendon is associated with inguinal-related groin pain: a MRI study. Eur Radiol. 2020;30:1517-24.
20. Tsutsumi M, Nimura A, Akita K. The gluteus medius tendon and its insertion sites: an anatomical study with possible implications for gluteus medius tears. J Bone Joint Surg Am. 2019;101:177-84.

21. Huang BK, Campos JC, Michael Peschka PG, et al. Injury of the gluteal aponeurotic fascia and proximal iliotibial band: anatomy, pathologic conditions, and MR imaging. Radiographics. 2013;33:1437-52.

22. Blankenbaker DG, Ullrick SR, Davis KW, De Smet AA, Haaland B, Fine JP. Correlation of MRI findings with clinical findings of trochanteric pain syndrome. Skeletal Radiol. 2008;37:903-9.

23. Bredella MA, Azevedo DC, Oliveira AL, et al. Pelvic morphology in ischiofemoral impingement. Skeletal Radiol. 2015;44:249-53.

24. López-Royo MP, Valero-Tena E, Roca M. Anatomical analysis of the pelvis to identify any predisposing anatomical factors for ischiofemoral space pathology: a retrospective study. Br J Radiol. 2020;93:20190556.

25. Vicentini JR, Martinez-Salazar EL, Simeone FJ, Bredella MA, Palmer WE, Torriani M. Kinematic MRI of ischiofemoral impingement. Skeletal Radiol. 2020; https://doi.org/10.1007/ s00256-020-03519-4.

26. Kheterpal AB, Harvey JP, Husseini JS, Martin SD, Torriani M, Bredella MA. Hip abductor tears in ischiofemoral impingement. Skeletal Radiol. 2020;49:1747-52.

27. Skalski MR, Matcuk GR, Patel DB, Tomasian A, White EA, Gross JS. Imaging coccygeal trauma and coccydynia. RadioGraphics. 2020;40:1090-106.

28. Rizkalla JM, Lines T, Nimmons S. Classifications in brief: the Denis classification of sacral fractures. Clin Orthop Relat Res. 2019;477:2178-81.

29. Hanna TN, Sadiq M, Ditkofsky N, et al. Sacrum and coccyx radiographs have limited clinical impact in the emergency department. Am J Roentgenol. 2016;206:681-6.

30. Mendel T, Ullrich BW, Hofmann GO, et al. Progressive instability of bilateral sacral fragility fractures in osteoporotic bone: a retrospective analysis of X-ray, CT, and MRI datasets from 78 cases. Eur J Trauma Emerg Surg. 2020; https://doi.org/10.1007/ s00068-020-01480-4.

Open Access This chapter is licensed under the terms of the Creative Commons Attribution 4.0 International License (http://creativecommons. org/licenses/by/4.0/), which permits use, sharing, adaptation, distribution and reproduction in any medium or format, as long as you give appropriate credit to the original author(s) and the source, provide a link to the Creative Commons license and indicate if changes were made.

The images or other third party material in this chapter are included in the chapter's Creative Commons license, unless indicated otherwise in a credit line to the material. If material is not included in the chapter's Creative Commons license and your intended use is not permitted by statutory regulation or exceeds the permitted use, you will need to obtain permission directly from the copyright holder. 